\title{
MEMBRANE SHELL EQUATION: CHARACTERIZATION OF THE SPACE OF SOLUTIONS*
}

\author{
Michel C. Delfour \\ Centre de recherches mathématiques, Université de Montréal \\ C. P. 6128, succ. centre-ville, Montréal QC, Canada H3C 3J7 \\ delfourCRM.UMontreal.CA
}

\begin{abstract}
The existence of solution to membrane shell equation is studied in a bounded open connected domain $\omega$ (Lipschitzian when $\omega$ has a boundary $\gamma$ ) in a $C^{1,1}$ midsurface for homogeneous Neumann boundary conditions or homogeneous Dirichlet boundary conditions on a part $\gamma_{0}$ of $\gamma$. It is proved that its tangential part is solution of the reduced membrane shell equation in $H^{1}(\omega)^{N}$ (resp. $H_{\gamma_{0}}^{1}(\omega)^{N}$ ) unique up to an element of a finite dimensional subspace, while its normal component belongs to a weighed $L^{2}(\omega)$ space by the pointwise norm of the second fundamental form. It is also shown that the reduced equation is equivalent to the equation for the projection onto the linear subspace of vector functions whose linear change of metric tensor is orthogonal to the second fundamental form of the midsurface.
\end{abstract}

\section{INTRODUCTION}

In recent papers $([8,9,11,12])$ it was established that the polynomial $P(2,1)$ model is both pertinent and basic in the theory of thin shells. It was shown in [8] that its solution converges to the solution of a coupled system of variational equations. For the plate and the bending dominated shell it yields (as the thickness $2 h$ goes to zero) the membrane shell equation and the asymptotic bending equation.

The first variational equation of the asymptotic coupled system coincides with the variational equation characterizing the asymptotic $P(0,1)$ model. It was shown in [8] that this equation decomposes into two equations: a first equation containing the Love-Kirchhoff group of terms and a second equation which coincides with the classical membrane shell equation. The detailed cor-

\footnotetext{
*This research has been supported by National Sciences and Engineering Research Council of Canada research grant A-8730 and by a FCAR grant (Ministère de l'Éducation du Québec).

The original version of this chapter was revised: The copyright line was incorrect. This has been corrected. The Erratum to this chapter is available at DOI: 10.1007/978-0-387-35359-3_40 
respondence with the covariant form of the classical membrane shell equation is given in [10]. The decomposition is achieved by variable elimination which results in the introduction of an effective compliance $C_{e P}$ associated with the initial three dimensional compliance $C$.

In this paper the membrane shell equation is studied in a bounded open connected domain $\omega$ (Lipschitzian when $\omega$ has a boundary $\gamma$ ) in a $C^{1,1}$ submanifold of codimension one for homogeneous Neumann boundary conditions or homogeneous Dirichlet boundary conditions on a part $\gamma_{0}$ of $\gamma$ when $\gamma_{0}$ has nonzero $H_{N-2}$ Hausdorff measure. This paper is a companion paper to [8] where the spaces of solution $E^{0}$ and $E^{P}$ corresponding to the respective asymptotic $P(0,1)$ model and the membrane shell equation are defined as completions of appropriate quotient spaces. It gives a complete characterization of the space $E^{P}$ without extra condition on the second fundamental form. Such a characterization is currently available for the plate and uniform strong elliptic shells in $[13,5,6,7]$. It also shows that we can always associate with the vector functions of the space $E^{P}$ a class of tangential components which turns out to be solutions of the reduced membrane shell equation. This reduced equation is also connected with a projection onto a linear subspace of elements of $E^{P}$ whose linear change of metric tensor is orthogonal to the second fundamental form. Another consequence of the characterization of $E^{P}$ is the fact that in the asymptotic convergence of the solution of the $P(2,1)$ model we now know that the tangential component of the displacement of the midsurface strongly converges in $H^{1}(\omega)^{N}$ and the normal component in a weighed $L^{2}(\omega)$ space by the pointwise norm of the second fundamental form. The characterization given in this paper and the one of $E^{01}$ given in [9] for the $P(2,1)$ model sharpen the abstract results of [8].

For $N=3$, this extends to arbitrary $D^{2} b$ the available existence of solutions obtained by $[1,3]$ for $g^{0}=0$, homogeneous Dirichlet boundary conditions on the whole boundary, the special constitutive law $C^{-1} \varepsilon=2 \mu \varepsilon+\lambda \operatorname{tr} \varepsilon I$ and the uniform ellipticity of the 2-dimensional $C^{2}$-midsurface $\omega$. However in the case of uniform elliptic shells uniqueness does not so far follows directly in an obvious way. The first existence and uniqueness result seems to be due to [13] under relatively strong conditions. For a domain $\omega$ with a $C^{3}$ boundary $\gamma$ in an analytic midsurface, the existence and uniqueness of solutions $\left(\hat{v}_{\Gamma}^{0}, \hat{v}_{n}^{0}\right)$ in $H_{0}^{1}(\omega)^{3} \times L^{2}(\omega)$ was established by $[6,7]$. The conditions were relaxed by $[1,3]$ : the midsurface is of class $C^{2}$ and the boundary $\gamma$ is Lipschitz for the existence (midsurface $C^{5}$ and the boundary $\gamma$ of class $C^{4}$ for existence and uniqueness).

Notation and Background Material. The inner product in $\mathbf{R}^{N}$ and the double inner product in $\mathcal{L}\left(\mathbf{R}^{N} ; \mathbf{R}^{N}\right)$ (space of $N \times N$ matrices or tensors) are denoted as

$$
x \cdot y=\sum_{i=1}^{N} x_{i} y_{i}, \quad A \cdot B=\sum_{i=1}^{N} \sum_{j=1}^{N} A_{i j} B_{i j} .
$$

${ }^{*} M$ denotes the transpose of of an arbitrary $k \times m$ matrix $M$.

In this paper the submanifold $\Gamma$ of codimension one is specified as the boundary of a subset $\Omega$ of $\mathbf{R}^{N}$. It is assumed that $\omega$ is a bounded open subset of $\Gamma$ 
and that $\Gamma$ is of class $C^{1,1}$ in a neighbourhood of $\omega$. This is equivalent to say that the algebraic distance function $b$ of $\Omega$ is $C^{1,1}$ in that neighborhood. Its gradient $\nabla b$ coincides with the unit exterior normal $n$ to $\Gamma$ on $\omega$ and its Hessian matrix $D^{2} b$ to the second fundamental form. Finally $P \stackrel{\text { def }}{=} I-n^{*} n$ will denote the orthogonal projection onto the tangent plane to $\omega\left(\left[n^{*} n\right]_{i j}=n_{i} n_{j}\right)$. For a detailed account of the intrinsic differential calculus on a $C^{1,1}$-submanifold, the reader is referred to the now available lecture notes [10, 8]. Finally it will be convenient to introduce the following notation for the decompositions of an $N \times N$ matrix $\tau$ into its tangential and normal parts along $\omega$

$$
\tau^{P} \stackrel{\text { def }}{=} P \tau P, \quad \tau_{n n} \stackrel{\text { def }}{=} \tau n \cdot n, \quad[t] \tau=\tau^{P}+(P \tau n)^{*} n+n^{*}(P \tau n)+\tau_{n n} n^{*} n
$$

and the spaces of symmetrical matrices

$$
\begin{gathered}
\operatorname{Sym}_{N} \stackrel{\text { def }}{=}\left\{\tau \in \mathcal{L}\left(\mathbf{R}^{N} ; \mathbf{R}^{N}\right):^{*} \tau=\tau\right\} \\
\operatorname{Sym}_{N}^{P} \stackrel{\text { def }}{=}\left\{\tau \in \operatorname{Sym}_{N}: \tau n=0\right\} \Leftrightarrow \forall \tau \in \operatorname{Sym}_{N}, \tau^{P} \in \operatorname{Sym}_{N}^{P} .
\end{gathered}
$$

\section{MEMBRANE SHELL EQUATION}

It was shown in [8] that the membrane shell equation can be obtained by decomposition of the variational equation of the asymptotic $P(1,0)$ model which also yields the typical group of terms occurring in the Love-Kirchhoff condition. It involves an effective compliance $C_{e P}$ which retains the properties of the three-dimensional compliance $C$. So for the purposes of this paper it is convenient to start with the following assumption on the effective compliance.

Assumption 2.1 The effective compliance is a linear bijective and symmetrical transformation of $\operatorname{Sym}_{N}^{P}$ such that there exists a constant $\alpha>0$ for which

$$
\forall \tau^{P} \in \operatorname{Sym}_{N}^{P}, \quad C_{e P}^{-1} \tau^{P} \cdot . \tau^{P} \geq \alpha\left\|\tau^{P}\right\|^{2}
$$

The membrane shell variational equation is given by: for all $v^{0} \in H^{1}(\omega)^{N}$

$$
\int_{\omega} C_{e P}^{-1} \varepsilon_{\Gamma}^{P}\left(\hat{v}^{0}\right) \cdot \varepsilon_{\Gamma}^{P}\left(v^{0}\right) d \Gamma=\ell^{P}\left(v^{0}\right)
$$

where the right-hand side is specified by a linear functional $\ell^{P}$. Associate with $\varepsilon_{\Gamma}^{P}$ the space

$$
V \stackrel{\text { def }}{=}\left\{v \in L^{2}(\omega)^{N}: v_{\Gamma} \in H^{1}(\omega)^{N}\right\} \subset H \stackrel{\text { def }}{=} L^{2}(\omega)^{N}
$$

and define $E^{P}$ as the completion of the quotient space $V / \operatorname{ker} \varepsilon_{\Gamma}^{P}$ with respect to the norm associated with the inner product

$$
\int_{\omega} \varepsilon_{\Gamma}^{P}(u) \cdot \cdot \varepsilon_{\Gamma}^{P}(v) d \Gamma
$$


Similarly for homogeneous Dirichlet boundary conditions on a part $\gamma_{0}$ of $\gamma$, denote by $E_{\gamma_{0}}^{P}$ the completion of the quotient space

$$
V_{\gamma_{0}} / \operatorname{ker} \varepsilon^{P}, \quad V_{\gamma_{0}} \stackrel{\text { def }}{=}\left\{v \in L^{2}(\omega)^{N}: v_{\Gamma} \in H_{\gamma_{0}}^{1}(\omega)^{N}\right\}
$$

with respect to the norm generated by the scalar product (1.3). By Assumption 2.1 on $C_{e P}$, the bilinear term in $(1.1)$ is continuous and coercive in $E^{P}$.

Theorem 2.2 Let Assumption 2.1 on $C_{e P}$ be verified.

(i) Given $\ell^{P} \in\left(E^{P}\right)^{\prime}$, that is there exists $c>0$ such that for all $v^{0} \in H^{1}(\omega)^{N}$

$$
\left|\ell^{P}\left(v^{0}\right)\right| \leq c\left\|\varepsilon_{\Gamma}^{P}\left(v^{0}\right)\right\|_{L^{2}(\omega)}
$$

the variational equation: to find $\hat{v}^{0} \in E^{P}$ such that for all $v^{0} \in H^{1}(\omega)^{N}$

$$
\int_{\omega}\left[C_{e P}^{-1} \varepsilon_{\Gamma}^{P}\left(\hat{v}^{0}\right)\right] \cdot \cdot \varepsilon_{\Gamma}^{P}\left(v^{0}\right) d \Gamma=\ell^{P}\left(v^{0}\right)
$$

has a unique solution $\hat{v}^{0}$ in $E^{P}$.

(ii) Assume that $\omega$ is connected and that $\gamma_{0}$ is a subset of $\gamma$ with strictly positive $H_{N-2}$ measure. Given $\ell^{P} \in\left(E_{\gamma_{0}}^{P}\right)^{\prime}$, that is there exists $c>0$ such that for all $v^{0} \in H_{\gamma_{0}}^{1}(\omega)^{N}$

$$
\left|\ell^{P}\left(v^{0}\right)\right| \leq c\left\|\varepsilon_{\Gamma}^{P}\left(v^{0}\right)\right\|_{L^{2}(\omega)}
$$

the variational equation: to find $\hat{v}^{0} \in E_{\gamma_{0}}^{P}$ such that for all $v^{0} \in H_{\gamma_{0}}^{1}(\omega)^{N}$

$$
\int_{\omega}\left[C_{e P}^{-1} \varepsilon_{\Gamma}^{P}\left(\hat{v}^{0}\right)\right] \cdot \varepsilon_{\Gamma}^{P}\left(v^{0}\right) d \Gamma=\ell^{P}\left(v^{0}\right)
$$

has a unique solution $\hat{v}^{0}$ in $E_{\gamma_{0}}^{P}$.

\section{REDUCED MEMBRANE SHELL EQUATION}

The membrane shell equation can be further decomposed into a system of two equations. For test functions $v \in V$, that is $\left(v_{\Gamma}^{0}, v_{n}^{0}\right) \in H^{1}(\omega)^{N} \times L^{2}(\omega)$,

$$
\begin{gathered}
\varepsilon_{\Gamma}^{P}\left(v^{0}\right)=\varepsilon_{\Gamma}^{P}\left(v_{\Gamma}^{0}\right)+v_{n}^{0} D^{2} b \\
\exists c>0, \forall v_{n}^{0} \in H^{1}(\omega), \quad\left|\ell^{P}\left(v_{n}^{0} n\right)\right| \leq c\left\|v_{n}^{0} D^{2} b\right\|_{L^{2}(\omega)} \leq c^{\prime}\left\|v_{n}^{0}\right\|_{L^{2}(\omega)} \\
\Rightarrow \exists f^{P} \in L^{2}(\omega) \text { such that } \ell^{P}\left(v_{n}^{0} n\right)=\int_{\omega} f^{P} v_{n}^{0} d \Gamma
\end{gathered}
$$

It will be convenient to define the function

$$
f_{n}^{P}(X) \stackrel{\text { def }}{=} \begin{cases}f^{P}(X) /\left\|D^{2} b(X)\right\|, & \text { if }\left\|D^{2} b(X)\right\| \neq 0 \\ 0, & \text { if }\left\|D^{2} b(X)\right\|=0\end{cases}
$$

By construction $f_{n}^{P}\left\|D^{2} b\right\| \in L^{2}(\omega)$. 
Denote by $H_{t}^{1}(\omega)^{N}$ (resp. $H_{\gamma_{0} t}^{1}(\omega)^{N}$ ) the subspace $\left\{v \in H^{1}(\omega)^{N}\right.$ (resp. $\left.\left.H_{\gamma_{0}}^{1}(\omega)^{N}\right): v \cdot n=0\right\}$ of tangential vectors. The decomposition yields the two equations

$$
\begin{gathered}
{\left[C_{e P}^{-1} \varepsilon_{\Gamma}^{P}\left(\hat{v}^{0}\right)\right] \cdot D^{2} b=f^{P}=f_{n}^{P}\left\|D^{2} b\right\|} \\
\forall v_{\Gamma}^{0} \in H_{t}^{1}(\omega)^{N}, \quad \int_{\omega}\left[C_{e P}^{-1} \varepsilon_{\Gamma}^{P}\left(\hat{v}^{0}\right)\right] \cdot \varepsilon_{\Gamma}^{P}\left(v_{\Gamma}^{0}\right) d \Gamma=\ell^{P}\left(v_{\Gamma}^{0}\right)
\end{gathered}
$$

where by condition (1.4) on $\ell^{P}$

$$
\exists c>0, \forall v_{\Gamma}^{0} \in H_{t}^{1}(\omega)^{N}, \quad\left|\ell^{P}\left(v_{\Gamma}^{0}\right)\right| \leq c\left\|\varepsilon_{\Gamma}^{P}\left(v_{\Gamma}^{0}\right)\right\|_{L^{2}(\omega)} .
$$

In the case of the plate $\left(D^{2} b=0\right), \varepsilon_{\Gamma}^{P}\left(v^{0}\right)=\varepsilon_{\Gamma}^{P}\left(v_{\Gamma}^{0}\right)+v_{n}^{0} D^{2} b=\varepsilon_{\Gamma}^{P}\left(v_{\Gamma}^{0}\right)$ and there is only the variational equation

$$
\forall v_{\Gamma}^{0} \in H^{1}(\omega)^{N}, \quad \int_{\omega}\left[C_{e P}^{-1} \varepsilon_{\Gamma}^{P}\left(\hat{v}_{\Gamma}^{0}\right)\right] \cdot \cdot \varepsilon_{\Gamma}^{P}\left(v_{\Gamma}^{0}\right) d \Gamma=\ell^{P}\left(v_{\Gamma}^{0}\right)
$$

which completely specifies $\hat{v}_{\Gamma}^{0} \in H_{t}^{1}(\omega)^{N} / \operatorname{ker} \varepsilon_{\Gamma}^{P}\left(\operatorname{resp} . H_{\gamma_{0} t}^{1}(\omega)^{N}\right)$ and $\hat{v}_{n}^{0}$ is arbitrary. There is a generalization of this result without adding new conditions on $D^{2} b$. The second equation (1.8) specifies the tangential part $\hat{v}_{\Gamma}^{0}$ of $\hat{v}^{0}$ up to an element of some appropriate equivalence class providing a natural decomposition of the membrane shell equation into an equation for the equivalence class of $\hat{v}_{\Gamma}^{0}$ and an equation for $\hat{v}_{n}^{0}$ again modulo another equivalence class. In the case of the plate the corresponding equivalence class for $\hat{v}_{n}^{0}$ is so big that there is no information on $\hat{v}_{n}^{0}$ and we have uniqueness for $\hat{v}_{\Gamma}^{0}$ in the case of homogeneous Dirichlet boundary conditions on a part of the boundary.

Denote by $[v]_{E}$ the equivalence class of $v$ in $E^{P}$ (resp. $E_{\gamma_{0}}^{P}$ ). Let

$$
\omega_{0} \stackrel{\text { def }}{=}\left\{x \in \omega: D^{2} b(x)=0\right\} \text { and } \omega_{+} \stackrel{\text { def }}{=} \omega \backslash \omega_{0} .
$$

For $v \in V$ (resp. $\left.V_{\gamma_{0}}\right)$ define the function

$$
\pi_{S}(v) \stackrel{\text { def }}{=} \begin{cases}v-\frac{C_{e P}^{-1} \varepsilon_{\Gamma}^{P}(v) \cdot D^{2} b}{C_{e P}^{-1} D^{2} b \cdot D^{2} b} n, & \text { in } \omega_{+} \\ v, & \text { in } \omega_{0}\end{cases}
$$

Using the identity $\varepsilon_{\Gamma}^{P}(v)=\varepsilon_{\Gamma}^{P}\left(v_{\Gamma}\right)+v_{n} D^{2} b$, it is easy to verify that for all $v \in V\left(\right.$ resp. $\left.V_{\gamma_{0}}\right)$

$$
\varepsilon_{\Gamma}^{P}\left(\pi_{S}(v)\right)= \begin{cases}\varepsilon_{\Gamma}^{P}(v)-\frac{C_{e P}^{-1} \varepsilon_{\Gamma}^{P}(v) \cdot D^{2} b}{C_{e P}^{-1} D^{2} b \cdot D^{2} b} D^{2} b, & \text { in } \omega_{+} \\ \varepsilon_{\Gamma}^{P}(v), & \text { in } \omega_{0}\end{cases}
$$

For each $v_{\Gamma} \in H_{t}^{1}(\omega)^{N}$ (resp. $H_{\gamma_{0} t}^{1}(\omega)^{N}$ ) define the tensor

$$
\tilde{\varepsilon}_{\Gamma}^{P}\left(v_{\Gamma}\right) \stackrel{\text { def }}{=} \varepsilon_{\Gamma}^{P}\left(\pi_{S}\left(v_{\Gamma}\right)\right)= \begin{cases}\varepsilon_{\Gamma}^{P}\left(v_{\Gamma}\right)-\frac{C_{e P}^{-1} \varepsilon_{\Gamma}^{P}\left(v_{\Gamma}\right) \cdot D^{2} b}{C_{e P}^{-1} D^{2} b \cdot D^{2} b} D^{2} b, & \text { in } \omega_{+} \\ \varepsilon_{\Gamma}^{P}\left(v_{\Gamma}\right), & \text { in } \omega_{0}\end{cases}
$$

the quotient space

$$
V^{P} \stackrel{\text { def }}{=} H_{t}^{1}(\omega)^{N} / \operatorname{ker} \tilde{\varepsilon}_{\Gamma}^{P} \quad\left(\operatorname{resp} . V_{\gamma_{0}}^{P} \stackrel{\text { def }}{=} H_{\gamma_{0} t}^{1}(\omega)^{N} / \operatorname{ker} \tilde{\varepsilon}_{\Gamma}^{P}\right)
$$


and the space

$$
U \stackrel{\text { def }}{=} \pi_{S}\left(H_{t}^{1}(\omega)^{N}\right) \quad\left(\text { resp. } U_{\gamma_{0}} \stackrel{\text { def }}{=} \pi_{S}\left(H_{\gamma_{0} t}^{1}(\omega)^{N}\right)\right)
$$

Consider the reduced membrane shell equation: to find $v_{\Gamma} \in V^{P}$ (resp. $V_{\gamma_{0}}^{P}$ ) such that for all $w_{\Gamma} \in H_{t}^{1}(\omega)^{N}$ (resp. $\left.H_{\gamma_{0} t}^{1}(\omega)^{N}\right)$

$$
\int_{\omega} C_{e P}^{-1} \tilde{\varepsilon}_{\Gamma}^{P}\left(v_{\Gamma}\right) \cdot \cdot \tilde{\varepsilon}_{\Gamma}^{P}\left(w_{\Gamma}\right) d \Gamma=\ell^{P}\left(\pi_{S}\left(w_{\Gamma}\right)\right)
$$

By condition (1.4) on $\ell^{P}$ there exists $c>0$ such that for all $w_{\Gamma} \in H_{t}^{1}(\omega)^{N}$

$$
\mid \ell^{P}\left(\pi_{S}\left(w_{\Gamma}\right) \mid \leq c\left\|\varepsilon_{\Gamma}^{P}\left(\pi_{S}\left(w_{\Gamma}\right)\right)\right\|_{L^{2}(\omega)}=c\left\|\tilde{\varepsilon}_{\Gamma}^{P}\left(w_{\Gamma}\right)\right\|_{L^{2}(\omega)}\right.
$$

and equation (1.14) has a unique solution in the completion of the quotient space $V^{P}=V / \operatorname{ker} \tilde{\varepsilon}_{\Gamma}^{P}$ with respect to the topology generated by the norm $\left\|\tilde{\varepsilon}_{\Gamma}^{P}\left(v_{\Gamma}\right)\right\|_{L^{2}(\omega)}$. We now give a sharper existence theorem for the reduced membrane shell equation and the membrane shell equation. This theorem is based on a characterization of the elements of the spaces $E^{P}$ and $E_{\gamma_{0}}^{P}$.

Theorem 3.1 Let Assumption 2.1 on $C_{e P}$ and (1.4) on $\ell^{P}$ be verified.

(i) There exists a solution $\hat{v}_{\Gamma}$ in $H_{t}^{1}(\omega)^{N}$ (resp. $\left.H_{\gamma_{0} t}^{1}(\omega)^{N}\right)$ to the reduced membrane shell equation (1.14) unique up to an element of $\operatorname{ker} \tilde{\varepsilon}_{\Gamma}^{P}$ and

$$
\left[\pi_{S}\left(\hat{v}_{\Gamma}\right)\right]_{E}=\left[\pi_{S}(\hat{v})\right]_{E}
$$

where $[\hat{v}]_{E}$ is the solution of the membrane shell equation (1.5) (resp. (1.7)) in $E^{P}$ (resp. $\left.E_{\gamma_{0}}^{P}\right)$.

(ii) There exists a solution $\hat{u}$ such that $\hat{u}_{\Gamma} \in H_{t}^{1}(\omega)^{N}\left(\right.$ resp. $\left.H_{\gamma_{0} t}^{1}(\omega)^{N}\right)$ and $\hat{u}_{n}\left\|D^{2} b\right\| \in L^{2}(\omega)$ to the membrane shell equation (1.5) (resp. (1.7)) which is unique up to an element of $\operatorname{ker} \varepsilon_{\Gamma}^{P}$.

(iii) $\operatorname{ker} \tilde{\varepsilon}_{\Gamma}^{P}$ is finite dimensional. When $D^{2} b \neq 0$ almost everywhere in $\omega$, $\operatorname{ker} \varepsilon_{\Gamma}^{P}$ is also finite dimensional and

$$
\operatorname{ker} \varepsilon_{\Gamma}^{P}=\left\{v: v_{\Gamma} \in \operatorname{ker} \tilde{\varepsilon}_{\Gamma}^{P} \text { and } v_{n}=-\frac{C_{e P}^{-1} \varepsilon_{\Gamma}^{P}\left(v_{\Gamma}\right) \cdot D^{2} b}{C_{e P}^{-1} D^{2} b \cdot D^{2} b}\right\}
$$

This theorem necessitates the following theorem on the structure of the spaces spaces $E^{P}$ and $E_{\gamma_{0}}^{P}$ which follows from a sequence of lemmas. The proofs will be omitted for lack of space and will be given in a subsequent paper.

Theorem 3.2 Let Assumption 2.1 on $C_{e P}$ be verified.

(i) $\operatorname{ker} \tilde{\varepsilon}_{\Gamma}^{P}$ is finite dimensional and the space $V^{P}=V / \operatorname{ker} \tilde{\varepsilon}_{\Gamma}^{P}$ (resp. $V_{\gamma_{0}}^{P}=$ $\left.V_{\gamma_{0}} / \operatorname{ker} \tilde{\varepsilon}_{\Gamma}^{P}\right)$ is complete for the norm $\left\|\tilde{\varepsilon}_{\Gamma}^{P}\left(v_{\Gamma}\right)\right\|_{L^{2}(\omega)}$. 
(ii) The space $E^{P}$ (resp. $E_{\gamma_{0}}^{P}$ ) is equal to

$$
\left\{\begin{array}{ll}
u_{\Gamma}+u_{n} n: & u_{\Gamma} \in H_{t}^{1}(\omega)^{N}\left(\text { resp. } H_{\gamma_{0} t}^{1}(\omega)^{N}\right) \\
\text { and } u_{n}\left\|D^{2} b\right\| \in L^{2}(\omega)
\end{array}\right\} / \operatorname{ker} \varepsilon_{\Gamma}^{P}(1.17)
$$

Specifically for each $[v]_{E} \in E^{P}$, there exists a unique $\left[v_{\Gamma}\right]_{V} \in V^{P}=$ $H_{t}^{1}(\omega)^{N} / \operatorname{ker} \tilde{\varepsilon}_{\Gamma}^{P}\left(\operatorname{resp} . V_{\gamma_{0}}^{P}=H_{\gamma_{0} t}^{1}(\omega)^{N} / \operatorname{ker} \tilde{\varepsilon}_{\Gamma}^{P}\right)$ such that

$$
\left[\pi_{S}\left(u_{\Gamma}\right)\right]_{E}=\left[\pi_{S}(v)\right]_{E}
$$

and for each $u_{\Gamma}$ in the equivalence class $\left[v_{\Gamma}\right]_{V}$ the normal component

$$
u_{n} \stackrel{\text { def }}{=} \begin{cases}\frac{C_{e P}^{-1} \varepsilon_{\Gamma}^{P}\left(v-u_{\Gamma}\right) \cdot D^{2} b}{C_{e P}^{-1} D^{2} b \cdot D^{2} b}, & \text { in } \omega_{0} \\ 0, & \text { in } \omega_{+}\end{cases}
$$

is such that $u_{n}\left\|D^{2} b\right\| \in L^{2}(\omega)$ and

$$
\left[u_{\Gamma}+u_{n} n\right]_{E}=[v]_{E} .
$$

Conversely for all $u_{\Gamma} \in H_{t}^{1}(\omega)^{N}$ and $u_{n}\left\|D^{2} b\right\| \in L^{2}(\omega)$

$$
\left[u_{\Gamma}+u_{n} n\right]_{E} \in E^{P} \text {. }
$$

(iii) When $D^{2} b \neq 0$ almost everywhere in $\omega$, then $\operatorname{ker} \varepsilon_{\Gamma}^{P}$ is finite dimensional. Define the closed linear subspace

$$
\begin{gathered}
S^{P} \stackrel{\text { def }}{=}\left\{v \in E^{P}: C_{e P}^{-1} \varepsilon_{\Gamma}^{P}(v) \cdot D^{2} b=0\right\} \\
\left(\operatorname{resp} S_{\gamma_{0}}^{P} \stackrel{\text { def }}{=}\left\{v \in E_{\gamma_{0}}^{P}: C_{e P}^{-1} \varepsilon_{\Gamma}^{P}(v) \cdot D^{2} b=0\right\}\right)
\end{gathered}
$$

of $E^{P}$ (resp. $E_{\gamma_{0}}^{P}$ ). We first make sense of the map $\pi_{S}$ on $E^{P}$.

Lemma 3.3 The map

$$
[v]_{E} \mapsto \pi_{S}\left([v]_{E}\right) \stackrel{\text { def }}{=}\left[\pi_{S}(v)\right]_{E}: E^{P} \rightarrow S^{P}
$$

is well-defined, linear and continous. Moreover

$$
\begin{array}{r}
\overline{\pi_{S}(V) / \operatorname{ker} \varepsilon_{\Gamma}^{P}} E^{P}=\overline{\pi_{S}\left(V / \operatorname{ker} \varepsilon_{\Gamma}^{P}\right)} E^{P}=S^{P} \\
\forall v \in S^{P}, \quad \varepsilon_{\Gamma}^{P}\left(\pi_{S}(v)\right)=\varepsilon_{\Gamma}^{P}(v) .
\end{array}
$$

and $\pi_{S}$ is a projection, that is $\left[\pi_{S}(v)\right]_{E}=\left[\pi_{S}\left(\pi_{S}(v)\right)\right]_{E}$ in $E^{P}$.

Lemma 3.4 The map $\pi_{S}: H_{t}^{1}(\omega)^{N} \rightarrow U$ is a continuous linear bijection and $U$ is closed for the topology generated by the norm

$$
\|u\|_{U}=\left\{\left\|\varepsilon_{\Gamma}^{P}(u)\right\|_{L^{2}(\omega)}^{2}+\left\|u_{\Gamma}\right\|_{L^{2}(\omega)}^{2}+\left\|u_{n}\right\| D^{2} b\|\|_{L^{2}(\omega)}^{2}\right\}^{1 / 2}
$$


The space $V^{P}$ is complete for the norm $\left\|\tilde{\varepsilon}_{\Gamma}^{P}\left(v_{\Gamma}\right)\right\|_{L^{2}(\omega)}$ and $\operatorname{ker} \tilde{\varepsilon}_{\Gamma}^{P}$ is finite dimensional.

Lemma 3.5 The map

$$
\begin{aligned}
{\left[v_{\Gamma}\right]_{V} \mapsto \quad \pi_{S}\left(\left[v_{\Gamma}\right]_{V}\right) \stackrel{\text { def }}{=}\left[\pi_{S}(v)\right]_{E} } \\
: V^{P}=H_{t}^{1}(\omega)^{N} / \operatorname{ker} \tilde{\varepsilon}_{\Gamma}^{P} \rightarrow U / \operatorname{ker} \varepsilon_{\Gamma}^{P}
\end{aligned}
$$

is a well-defined isomorphism, where $[v]_{V}$ denotes the equivalence class of $v$ in $V^{P}, V^{P}$ is endowed with the topology generated by the norm $\left\|\tilde{\varepsilon}_{\Gamma}^{P}\left(v_{\Gamma}\right)\right\|$ and $U / \operatorname{ker} \varepsilon_{\Gamma}^{P}$ by the norm $\left\|\varepsilon_{\Gamma}^{P}(v)\right\|$ on $E^{P}$. Moreover

$$
S^{P}=U / \operatorname{ker} \varepsilon_{\Gamma}^{P}=\pi_{S}\left(H_{t}^{1}(\omega)^{N}\right) / \operatorname{ker} \varepsilon_{\Gamma}^{P}=\pi_{S}\left(V^{P}\right) .
$$

Lemma 3.6 For each $v \in E^{P}$, the projection $\left[\pi_{S}(v)\right]_{E}$ is the unique solution in $S^{P}$ of the variational equation: for all $w \in H^{1}(\omega)^{N}$

$$
\int_{\omega} C_{e P}^{-1} \varepsilon_{\Gamma}^{P}\left(\pi_{S}(v)-v\right) \cdot \cdot \varepsilon_{\Gamma}^{P}(w) d \Gamma=0
$$

and there is a solution $v_{\Gamma} \in H_{t}^{1}(\omega)^{N}$ unique up to an element of $\operatorname{ker} \tilde{\varepsilon}_{\Gamma}^{P}$ to the variational equation: for all $w_{\Gamma}$ in $H_{t}^{1}(\omega)^{N}$

$$
\int_{\omega} C_{e P}^{-1} \tilde{\varepsilon}_{\Gamma}^{P}\left(v_{\Gamma}\right) \cdot \tilde{\varepsilon}_{\Gamma}^{P}\left(w_{\Gamma}\right) d \Gamma=\int_{\omega} C_{e P}^{-1} \varepsilon_{\Gamma}^{P}(v) \cdot \cdot \varepsilon_{\Gamma}^{P}\left(\pi_{S}\left(w_{\Gamma}\right)\right) d \Gamma .
$$

Moreover

$$
\left[\pi_{S}(v)\right]_{E}=\left[\pi_{S}\left(v_{\Gamma}\right)\right]_{E}
$$

All this remains true for Dirichlet boundary conditions on a part $\gamma_{0}$ of the boundary with $H_{\gamma_{0} t}^{1}(\omega)^{N}$ in place of $H_{t}^{1}(\omega)^{N}$ and $E_{\gamma_{0}}^{P}$ in place of $E^{P}$.

Lemma 3.7 (i) The space $E^{P}$ is equal to

$$
\left\{u_{\Gamma}+u_{n} n: u_{\Gamma} \in H_{t}^{1}(\omega)^{N} \text { and } u_{n}\left\|D^{2} b\right\| \in L^{2}(\omega)\right\} / \operatorname{ker} \varepsilon_{\Gamma}^{P} \text { (1.27) }
$$

Specifically for each $[v]_{E} \in E^{P}$, there exists a unique $\left[v_{\Gamma}\right]_{V} \in V^{P}=$ $H_{t}^{1}(\omega)^{N} / \operatorname{ker} \tilde{\varepsilon}_{\Gamma}^{P}\left(\operatorname{resp} . V_{\gamma_{0}}^{P}=H_{\gamma_{0} t}^{1}(\omega)^{N} / \operatorname{ker} \tilde{\varepsilon}_{\Gamma}^{P}\right)$ such that

$$
\left[\pi_{S}\left(v_{\Gamma}\right)\right]_{E}=\left[\pi_{S}(v)\right]_{E}
$$

and for each $u_{\Gamma}$ in the equivalence class $\left[u_{\Gamma}\right]_{V}$ the normal component

$$
u_{n} \stackrel{\operatorname{def}}{=} \begin{cases}\frac{C_{e P}^{-1} \varepsilon_{\Gamma}^{P}\left(\hat{v}^{0}-u_{\Gamma}\right) \cdot D^{2} b}{C_{e P}^{-1} D^{2} b \cdot D^{2} b}, & \text { in } \omega_{0} \\ 0, & \text { in } \omega_{+}\end{cases}
$$

is such that $u_{n}\left\|D^{2} b\right\| \in L^{2}(\omega)$ and

$$
\left[u_{\Gamma}+u_{n} n\right]_{E}=[v]_{E} .
$$




$$
\begin{gathered}
\text { Conversely for all } u_{\Gamma} \in H_{t}^{1}(\omega)^{N} \text { and } u_{n}\left\|D^{2} b\right\| \in L^{2}(\omega) \\
{\left[u_{\Gamma}+u_{n} n\right]_{E} \in E^{P} .}
\end{gathered}
$$

(ii) When $D^{2} b \neq 0$ almost everywhere in $\omega$, then $\operatorname{ker} \varepsilon_{\Gamma}^{P}$ is finite dimensional. The lemma remains true for Dirichlet boundary conditions on a part $\gamma_{0}$ of the boundary with $H_{t, \gamma_{0}}^{1}(\omega)^{N}$ and $E_{\gamma_{0}}^{P}$ in place of $H_{t}^{1}(\omega)^{N}$ and $H_{\gamma_{0} t}^{1}(\omega)^{N}$.

\section{References}

[1] Ph. G. Ciarlet and V. Lods, Ellipticité des équations membranaires d'une coque uniformément elliptique, C. R. Acad. Sci. Paris Sér. I Math. 318 (1994), 195-200.

[2] -, Analyse asymptotique des coques linéairement élastique. I. Coques membranaires, C. R. Acad. Sci. Paris Sér. I Math. 318 (1994), 863-868.

[3] -, On the ellipticity of linear membrane shell equations, J. Math. Pures Appl. 75 (1996), 107-124.

[4] -, Asymptotic analysis of linearly elastic shells. Generalized membrane shells, J. Elasticity 43 (1996), 147-188.

[5] -, Asymptotic analysis of linearly elastic shells. I. Justification of membrane shell equations, Arch. Rational Mech. Anal. 136 (1996), 116-161.

[6] Ph. G. Ciarlet and É. Sanchez-Palencia, Un théorème d'existence et d'unicité pour les équations des coques membranaires, C. R. Acad. Sci. Paris Sér. I Math. 317 (1993), 801-805.

[7] -, An existence and uniqueness theorem for the two-dimensional linear membrane shell equations, J. Math. Pures Appl. 75 (1996), 51-67.

[8] M.C. Delfour, Intrinsic differential geometric methods in the asymptotic analysis of linear thin shells, Boundaries, interfaces and transitions (M.C. Delfour, ed.), CRM Proc. Lecture Notes, Amer. Math. Soc., Providence, RI, 1998, pp. 19-90.

[9] -, Intrinsic $P(2,1)$ thin shell model and Naghdi's models without a priori assumption on the stress tensor, in Proc International Conference on Optimal Control of Partial Differential Equations, K.H. Hoffmann, G. Leugering, F. Tröltzsch , eds., Birkhäuser, to appear.

[10] M.C. Delfour and J.-P. Zolésio, Differential equations for linear shells: comparison between intrinsic and classical models, Advances in Mathematical SciencesCRM's 25 years (Luc Vinet, ed.), CRM Proc. Lecture Notes, Amer. Math. Soc., Providence, RI, 1997, pp. 42-124.

[11] - Convergence to the asymptotic model for linear thin shells, Optimization Methods in Partial Differential Equations (S. Cox and I. Lasiecka, eds.), Contemp. Math., vol. 209, AMS, Providence, RI, 1977.

[12] - Convergence of the linear $P(1,1)$ and $P(2,1)$ thin shells to asymptotic shells, Proc. Plates and Shells: from theory to practice (M. Fortin, ed.), CRM Proc. Lect. Notes ser., Amer. Math. Soc., Providence, RI, 1977, to appear

[13] Ph. Destuynder, Sur la justification des modèles de plaques et de coques par les méthodes asymptotiques, Doctoral dissertation, Univ. Pierre et Marie Curie, 1980.

[14] É. Sanchez-Palencia, Statique et dynamique des coques minces. I. Cas de flexion pure non inhibée, C. R. Acad. Sc. Paris Sér. I Math. 309 (1989), 411-417.

[15] - Statique et dynamique des coques minces. II. Cas de flexion pure inhibée - Approximation membranaire, C. R. Acad. Sc. Paris Sér. I Math. 309 (1989), 531-537. 